\title{
The Basic Path of Improving the Two Levels of the Administrative Law Enforcement Accountability System in Cities and Counties
}

\author{
Jianping Miao
}

The County Economic Research Center of Economics and Management School of Neijiang Normal University, Neijiang, Sichuan, 641100, China

113597987@qq.com

Keywords: Cities and counties; Administrative law enforcement accountability; System; Path

Abstract. The in-depth development of the construction of "the two levels of the administrative law enforcement supervision and accountability system in cities and counties" is an urgent requirement to strengthen the supervision of the administrative law enforcement of the two levels of governments in city and county and promote the law-based administration of the two levels of governments in city and county, and is the necessity of promoting the law-based administration of local government and building the law-based government, which is of great practical guiding significance. The improvement of the administrative law enforcement accountability system needs the rule of law of administrative law enforcement accountability, needs the clear definition of the scope of the subject who undertakes responsibility for administrative law enforcement, and needs to master the key control points of the establishment of the administrative law enforcement accountability system.

Acknowledgements: This paper is one of the staged achievements of the key project "Research on the Mode and Mechanism of Social Organizations Participating in Community Home Care Service" (17SA0064) of the Sichuan Provincial Department of Education.

\section{完善市县两级行政执法责任追究机制的基本路径}

\author{
苗建萍 \\ 内江师范学院经济与管理学院县域经济研究中心，内江 中国，641100
}

113597987@qq.com

摘要: 深入开展 “市县两级行政执法监督及责任追究机制”建设是加强市县两级政府行政执法监督，促进市县两级政府 依法行政的迫切要求，是推动地方政府依法行政，构建法治政府的必要，具有极强现实指导意义。行政执法责任追究机制的 完善需要行政执法问责的法治化，行政执法责任承担主体范围的明确界定，以及掌握行政执法责任追究机制构建的关键控制 点。

关键词: 市县; 行政执法责任追究; 机制；路径

1 引言

实现法治是人类治理社会的理想模式，而法治化正是我们向这一宏伟蓝图探索的过程，“法治化是法 治理念向法治现实转变的过程, 也是人治实践向法治实践过渡转型的过程。”由于我国的社会形态与历史 发展中包含了很多特殊性, 将我国行政执法问责机制中最核心的技术和方法上升到法律和制度的层面, 特 别是用程序来保证问责沿着法治的轨道进行，这不仅是法律回应问责机制在我国发展过程中现实需求的生 动表现，更是问责从当前实践困境中得以解脱的根本出路。 


\section{2 行政执法问责的法治化: 行政问责的必然选择}

“法律是主权者意志的体现, 是众人智慧的结晶, 是国家公共权力赖以建立和运行的准则和根据。” 法律本身所蕴含的道德性以及至高无上的权威性, 能够充分发挥其对行政权力乃至政治权力的约束以及对 公民自由权利的保障功能。正如博登海默所说的 “法律的基本作用之一乃是约束和限制权力, 而不论这种 权力是私人权力还是政府权力。在法律统治的地方, 权力的自由行使受到了规则的阻碍, 这些规则迫使掌 权者按照一定行为方式行事。通过颁布旨在指导未来行动的行为标准, 法律缩小了就事论事的判决的范围。 与有着几百年法治基础和传统的西方国家相比, 我国目前尚不具备肥沃的宪政土壤, 尤其在我国这样一个 依旧有着浓厚 “官本位” 思想的社会里。正如有学者认为, 中国传统政治文化的核心就是官本位, 具体表 现在三个方面: 一是万事皆下品, 唯有做官高; 二是一人得道, 鸡犬升天; 三是官进则荣, 官退则耻。而 “问责制” 自 2002 年正式引入中国大陆以来, 虽已发展为我国为官的政治逻辑, 但是在实践的运行和发 展过程中遇到种种阻力, 需要我们借助外力的作用强制发展并完善诸如西方国家为确保问责的良性运行所 需要的内在条件、运行条件以及外部环境的建设。

实现对行政执法问责的法律规制, 并以国家机器为坚强后盾, 并利用法律所应具有的内在道德性、强 制性以及权威性的特质, 一方面可以克服问责的盲目性和随意性, 使得行政执法人员被限制在适当的范围 内, 不致为他们所拥有的权力所诱惑, 利用他们本来不熟悉的或不愿承认的手段来行使权力, 同时可以利 用法律所蕴含的程序正义来抵消难以在实体层面对行政官员问责机制全方位控制的弊端。另一方面也可以 使人们知道他们的责任并在法律范围内得到安全和保障。

\section{3 行政执法责任承担主体范围界定: 实现行政执法问责的基本前提}

\section{1 行政执法责任承担主体的范围界定及细化}

课题组认为从立法的严肃性、准确性来说, 在立法中应该明确界定主要用语的范围, 否则将会产生歧 义从而不利于该法的执行。因此，在行政执法责任制法规规章中应明确行政执法责任承担主体的范围，从 而有利于行政执法责任的追究和承担。考察各地的立法, 我们发现, 我国目前地方行政执法责任制立法中 行政执法责任承担主体和行政主体界限不明、范围混淆, 且各地对行政执法责任承担主体细化的规定不统

一，缺乏承担行政执法责任主体的具体规定。

本研究将行政执法主体界定为: 行政机关和法律法规授权的组织和行政执法人员, 而受委托的组织和 行政执法人员由于不具有独立的行政主体资格，因此不能够确定为行政执法责任的承担主体。

所谓行政执法人员是国家行政机关依法录用或委托并赋予其相应执法权的工作人员。从我国现阶段 看, 行政执法人员主要有两种: 一种是当然的行政执法人员, 即行政机关中拥有执法权的正式在编人员和 法律、法规授权的执法组织中的人员；另一种是因受行政机关合法委托而获得执法权的组织中的人员。

在确定行政执法责任承担主体的范围后我们还要对行政执法责任承担主体做进一步的细化，区分不同 层次不同情况下相应的责任承担主体，这样才能使立法的规定具有实际操作性，使行政执法责任落到实处。

我们认为从有利于追究行政执法责任的角度来看, 应采取上海、江苏的立法模式即将行政执法责任承 担主体划分为直接责任人员和直接主管人员后再区分不同情况确定责任的承担主体。其中直接责任人员, 是指行政执法事项的具体承办人。直接主管人员，是指行政执法事项的审核人和批准人。审核人，包括行 政机关内设机构负责人、分管领导, 以及按规定行使审核职权的其他审核人。批准人, 包括签发行政决定 
的行政机关负责人, 以及按规定或者经授权行使批准职权的其他批准人。由于在行政管理过程中, 许多行 政行为的制定实施是经过集体决策、法定机构鉴定或者基于听证后而为的行为。其名义上虽是行政机关的 行为, 但在实际上并非是由该行政机关承担责任。因其存在着名实不符的情况, 所以根据其性质不同可分 为依据法律规定作出行为的责任承担以及无法定依据作出行政行为的责任承担两种情形:

一是依据法律规定作出行政行为, 产生行政过错的责任承担主体如按照法定要求和程序作出集体决策 而签发的, 根据现有实践情况来看, 决策人员就无承办人、审核人和批准人之分, 而是作为批准人的身份 来担责。但根据《公务员法》及相关法律规定, 有会议记录或其他材料能证明决策人有疑义或正确意见而 未被采纳的可予以免责。

在行政机关的层级监督中, 上级机关依法改变或撤销下级行政决定而导致行政过错行为发生的, 具体 的责任承担则由上级机关的直接责任人承担。对于法律、法规和规章规定实施行政行为应当进行听证的, 行政机关负责人采纳主持人的错误建议而导致行政过错的, 主持人将被视为审核人承担责任, 机关负责人 视为批准人来承担责任。这是因为我国目前一般是由行政机关内部人员来做主持人, 主持人在听证中有权 对该行为进行定性审核, 而且我国实行行政首长负责制, 正是因为其签发, 才产生了法律效力, 所以负责 人视为批准人来担责。与此相反, 机关负责人若未采纳听证主持人根据听证会所提出的正确建议而导致行 政过错的，该主持人因其已尽审核义务而免责，但是机关负责人还应视为批准人担责。

二是没有法定依据作出行政行为, 而产生行政过错的责任承担主体, 集体决策并非法定, 而是在行政 机关内部集体讨论后由主管或分管领导决定实施该行为而导致行政过错的, 该领导则按照批准人来担责, 其他参加讨论的人员作为审核人来担责。

而对于因领导干预而执行公务作出的行政行为致使行政相对人遭受一定法律后果或危害后果的, 承办 人员在依法向该领导提出改正或撤销意见, 而上级不改变或要求立即执行的, 执行的后果由领导负责, 承 办人免责。但工作人员执行明显违反决定或命令的, 应当依法承担相应的责任。上级机关非依照法定职权 而实施干预而导致行政过错结果发生的, 具体实施人来承担承办人的责任, 除非有充分的证据能证明上级 机关非依法定职权实施干预行为与该行为决定的结果有因果关系，同时，行政机关自行决定根据专门的鉴 定机构或者专家提供的论证结论为依据而导致行政过错的, 作出该行政行为的直接责任人员则必须按照其 在该行为实施过程中的相应地位来承担相应责任。

\section{2 “问” 的涵义及其基本要素}

对 “问” 字涵义的界定应当置于 “问责”一词的具体语境之中。2005 版的《现代汉语词典》将 “问责” 一词解释为责任追究, 并将 “问” 定义为 “追究。而根据《汉语大词典》对 “问” 的界定, 不仅有追究之 意, 而且还可以解释为 “询问”。《汉语大词典》对 “问” 的解释主要包括一是询问、诘问; 二是论难, 探讨; 三是考察, 过问; 四是审讯; 五是追究等等。这里我们认为 “问” 应该是一个动态的过程, 包括两 个方面的内容: 首先是问的主体对问的对象进行询问以及问的对象对问的主体解释说明的过程; 其次是问 责主体对问的对象进行责任追究的过程。完整意义上的问的过程需要涵盖问的主体、问的对象、问的范围、 问的程序、问的方式、问的执行以及问后的救济共七个方面的基本要素。

\section{3 “责” 的内涵}

所谓责, 即责任的简称。关于对责任内涵的解释, 中外学者发表了不同的见解, 可谓仁者见仁, 智者 见智。纵观学界对责任的界定, 目前已经在两个方面达成共识: 首先, 责任意味着责任主体应尽的职责和 
义务, 它要求责任主体不但要做正确的事, 还要正确的做事, 属于积极责任的范畴; 其次, 责任主体因未 履行或未正确履行职责和义务而承担的否定性后果, 即应当追究的责任, 属于消极责任的范畴。总之, “责 任”一次应当是其内部逻辑体系中所蕴含的积极责任与消极责任的有机统一体。

鉴于本研究旨在建构问责语境中行政执法人员的责任体系，将行政执法人员责任分为以下四种: 政治 责任、行政责任、法律责任和道德责任，这四类责任之间相互区别，又相互影响和渗透，共同形成一个多 元复合的责任体系。

\section{4 行政执法责任追究机制构建的关键控制点}

所谓问责机制说到底是一个 “谁来问责”、“向谁负责” 的问题。权力是人民赋予的，当然是要对人 民负责。所以问责机制的内驱力最终要落实在群众的监督权上。常态化的政府问责，必须以健全的问责机 制为基础，通过立法确保各级政府部门和官员的权力始终处于一种负责任状态，杜绝任何行使权力的行为 脱离法定责任机制的监控。

构建健全的行政执法责任追究机制必须在问责基本原则的指导和支撑下，分析并阐释问责语境中行政 执法人员责任的构成要件及行政执法责任追究机制构建的关键控制点。

行政执法人员责任的构成要件包括: 责任主体、过错、损害结果、因果关系; 行政执法责任追究机制 构建的关键控制点主要应明确: 问责主体、问责对象（责任主体）、问责范围、问责程序、问责的方式及 其适用、问责的监督、考核评议等。

\section{5 致谢}

本文为四川省教育厅重点项目《社会组织参与社区居家养老服务模式与及机制研究》(17SA0064) 的阶 段性成果之一。

\section{参考文献:}

[1] 司春燕．法治与法治化概念辨析 [J]．山东省农业管理干部学院学报，2009 年第 1 期.

[2] 胡旭晨，蒋先福，法理学 [M]．湖南人民大学出版社，2001 年版，第 377 页.

[3] [美]E. 博登海默. 法理学： 法律哲学与法律方法 [M]， 邓正来翻译， 中国政法大学出版社 1999 年版，第 358 页.

[4] 胡良俊. 议我国领导干部引处辞职制度 [J]。探讨. 2004 年第 12 期.

[5] 词典编辑室编. 现代汉语词典[M]. 商务印书馆. 2005 年版第 1431 页.

[6] 贤明. 政治责任与法律责任的比较分析 [J]. 政治学研究. 2000 年第 1 期．

[7] 林琼. 论行政责任在公共服务型政府中的实现 [J]. 湖南行政学院学报. 2005 年第 3 期.

\section{References}

[1] C.Y.Ci: Concept Discrimination between Rule of Law and Legalization[J], Journal of Shandong Agricultural Administrators' College, (2009)No.1.(In Chinese)

[2] X.C.Hu and X.F.Jiang: Jurisprudence[M], Hunan Renmin University Press, 2001, p.377.(In Chinese)

[3] (American) E. Bodenheimer: Jurisprudence: Legal Philosophy and Legal Methods[M], China University of Political Science and Law Press(1999), p.358.

[4] L.J.Hu: A Brief Discussion on the Blame-Taking Resignation of the Leading Cadres in China[J], Academic Research(2004)No.12.(In Chinese)

[5] Dictionary Editing Room of the Language Research Institute of the Chinese Academy of Social Sciences, The Contemporary Chinese Dictionary[M], The Commercial Press(2005), p.1431.(In Chinese)

[6] X.M.Zhang: The Comparative Analysis of Political Responsibility and Legal Responsibility[J], Cass Journal of Political Science(2000)No.1.(In Chinese)

[7] Q.Lin: Discussion on the Realization of the Administrative Responsibility in the Public Service Government[J], Journal of Hunan Administration Institute(2005)No.3.(In Chinese) 